\title{
Design of an Optical Water Pollution Sensor Using a Single-Layer Guided-Mode Resonance Filter
}

\author{
Edward SADER and Abdallah SAYYED-AHMAD* \\ Department of Physics, Birzeit University, Birzeit, Palestine \\ ${ }^{*}$ Corresponding author: Abdallah SSAYYED-AHMAD \\ E-mail: asayyeda@birzeit.edu

\begin{abstract}
The optical characteristics of a simple, planar, single layer, dielectric Mg-based guided mode resonance filter (GMRF) were investigated by means of rigorous-coupled wave analysis (RCWA). This filter has great potential for real-life applications, especially as bio- and environmental sensors. The structure of the proposed sensor is compact, and all of its layers can be grown in a single process. In this paper, we present results on the design of a water pollution sensor in the violet region of the visible spectrum. The spectral and angular sensitivities of the sensor for both the transverse electric (TE) and transverse magnetic (TM) polarization modes were estimated and compared for various regions in the violet spectrum. A spectral response characterized with a narrow bandwidth and low reflection side bands was realized by carrying out extensive parameter search and optimization. Optimal spectral and angular sensitivities were found for the sensor with a grating thickness of $100 \mathrm{~nm}$ in the TM polarized mode where we found them to be $100 \mathrm{~nm}$ and 40 degrees, per index refraction unit, respectively.
\end{abstract}

Keywords: Diffractive optics, guided waves, filters, sensors, spectral sensitivity, angular sensitivity

Citation: Edward SADER and Abdallah SAYYED-AHMAD, "Design of an Optical Water Pollution Sensor Using a Single-Layer Guided-Mode Resonance Filter,” Photonic Sensors, DOI: 10.1007/s13320-013-0105-8.

\section{Introduction}

The $\mathrm{MgF}_{2} / \mathrm{MgO}$ combination used in our Mg-based guided mode resonance filter (GMRF) structure has been widely used in many scientific and technological applications. One related notable application is its usage as an insulating film-matrix for magnetic granules $\mathrm{Fe}_{50} \mathrm{Co}_{50}$ and $\mathrm{Fe}_{50} \mathrm{Co}_{50} \mathrm{~B}_{20}$, leading to the large tunnel magneto-resistance effect that can be utilized for magnetic sensing [1]. Another important application is its use as an optimal buffer layer for the high- $T_{C}$ superconductor yttrium-barium copper oxide (YBCO) integration onto silicon [2].
GMRFs are simple structures composed of a diffraction grating and a waveguide. When phase-matching conditions involving the wave vectors of the incident, reflected and transmitted radiations are met, externally propagating waves can be coupled into guided modes by the grating. These guided modes are "leaky" due to the modulation in the refractive index of the waveguide. This causes the guided modes to slowly leak out from the waveguide and recombine with the incident radiation to establish a resonance $[3,4]$.

The interest in optimizing guided-mode resonance (GMR) structures has tremendously grown in the last two decades due to the importance

Received: 18 November 2012 / Revised version: 7 May 2013

(C) The Author(s) 2013. This article is published with open access at Springerlink.com 
of these devices as tunable filters [5-10], modulators $[11,12]$ and sensors [13-17]. The ultimate goal of optimization procedures, for all cases, is to obtain a sharp peak in the diffraction efficiency spectrum of waveguide gratings and maximize the sensor optical sensitivity with respect to changes in the refractive index of the analyte. The sensitivity of the sensor and the location of the narrow peak in the reflection spectrum depend on the incident angle, the light polarization and the refractive index of the superstrate (analyte). In view of these optical properties, GMR structures can be used in many different applications such as bio-sensing, optoelectronics and optical communications. In this paper, we discuss the design process of an Mg-based GMR structure that can be used as a water pollution optical sensor in the violet region of the visible spectrum. We also present the values obtained for structural parameters that maximize the sensitivity of the sensor through a systematic determination of the diffraction efficiency of the reflected waves. The optimal parameters determined are the grating thickness, duty cycle, pitch and waveguide thickness.

\subsection{Basic GMR filter structure}

The guided mode structure studied in this work is schematically displayed in Fig. 1. The core of the filter is the magnesium fluoride $\left(\mathrm{MgF}_{2}\right)$ substrate

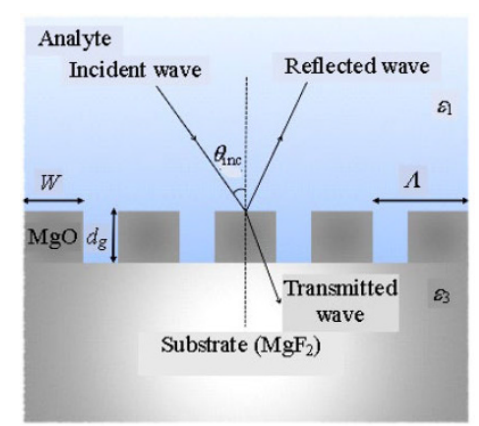

Fig. 1 Schematic structure of a single-layer Mg-based GMR sensor (the sensor is illuminated from the analyte side which is in contact with the periodic scattering grating structure, and the single layer planar structure facilitates the large scale production of such sensors). layer (region 3) which has a dielectric constant $\varepsilon_{3}$ and the magnesium oxide $(\mathrm{MgO})$ planar grating region which has a periodic structure with a period (pitch) $\Lambda$. In the latter region, the dielectric constant is spatially modulated as

$$
\varepsilon_{2}(x)=\varepsilon_{g}+\Delta \varepsilon \cos \left(\frac{2 \pi x}{\Lambda}\right)
$$

where $\varepsilon_{g}$ is the dielectric constant of the grating (region 2), which is the numerical average of the relative permittivity in the grating region, and $\Delta \varepsilon$ is its modulation amplitude. $\varepsilon_{1}$ is the dielectric constant of the analyte medium above the grating. This structure has the advantage of achieving high reflection efficiency without the need of a metallic coating, and thereby the absorption loss of the grating will be minimal.

\subsection{Theory of guided-mode resonance}

There are two basic interacting mechanisms that must be understood to fully explain the operation of a GMR. The first mechanism is diffraction of an incident wave by a grating. Inside the grating region, the amplitude of the propagating field has the same variations as that of the grating itself. When the grating is periodic, the electromagnetic fields can be expressed as a Fourier series where each term refers to a different diffracted order. The amplitude and phase of each diffracted mode must be calculated using Maxwell's equations, but the direction can be determined by the fundamental grating equation [18]:

$$
\sqrt{\varepsilon_{g}} \sin \theta_{m}=\sqrt{\varepsilon_{1}} \sin \theta_{\text {inc }}-m \frac{\lambda_{0}}{\Lambda}
$$

where $\theta_{\text {inc }}$ is the angle of incidence of the external wave, $\lambda_{0}$ is the free space wavelength, $m$ is an integer representing the order of the diffracted mode, and $\theta_{m}$ is the angle of the $m$ th diffracted mode inside the grating region.

The second mechanism is wave guiding where light is guided along a confined path by total internal reflection (TIR) [18]. Wave guiding can only occur when the effective index of the guided mode is 
greater than that of the surrounding media and less than that of the core itself. From this, a quantitative condition for wave guiding can be written as [3]

$$
\max \left\{\sqrt{\varepsilon_{1}}, \sqrt{\varepsilon_{3}}\right\} \leq\left|\frac{\beta_{m}}{k_{0}}\right| \leq \sqrt{\varepsilon_{g}}
$$

where $\beta_{m}$ is the propagation constant, $k_{0}$ is the effective index of refraction of the $m$ th-order guided mode, and $\varepsilon_{3}$ is the dielectric constant of the substrate (region 3 ). Exact values are not needed for $\beta_{m}$ as only limits on the effective index will be considered here. From a simple ray picture of a slab waveguide, a guided mode can be thought of as a light ray propagating at an angle $\theta_{m}$ such that

$$
\frac{\beta_{m}}{k_{0}}=\sqrt{\varepsilon_{g}} \sin \theta_{m} .
$$

A guided-mode resonance occurs when a diffracted order exists at the same angle as a guided mode. Guided modes can be related to the incident wave by substituting (4) into (2) and the new (2) into (3). This leads to an inequality that can be used to estimate regions where guided-mode resonances may occur. For pure water, the refractive index at $20{ }^{\circ} \mathrm{C}$ is 1.333 . We have demonstrated that our sensor is able to detect any small deviation from this reference value in the range of $1.32-1.37$.

\section{Methods}

In this study, the relatively fast and efficient rigorous coupled wave analysis (RCWA) algorithm [19] was utilized to solve Maxwell equations for the periodic diffracting GMR filter structure to obtain the diffraction efficiency for the reflected radiation for TM and TE modes. Since the accuracy of the solution obtained by RCWA depends on the number of terms retained in the space-harmonic expansion of the electromagnetic wave field, we systematically studied the convergence behavior of the solution as a function of the number of terms kept. We found out that for the filter structure used in this study using a cutoff of ten terms guaranteed convergence independently of the wavelength of the incident light, the thickness of the grating and the refractive index of the analyte. The simulations were performed using the optical grating solver multilayer rigorous coupled wave analysis (MRCWA) [20]. MRCWA is flexible and allows the study of the arbitrary grating profiles, depth, and permittivity. It allows simulating structures composed of both dielectric and conducting materials. In this study, the refractive index profiles as a function of the radiation wavelength for $\mathrm{MgO}$ and $\mathrm{MgF}_{2}$ were extracted from the Filmetrics index of refraction database [21].

\section{Results and discussion}

\subsection{Variation of the diffraction efficiency with the grating thickness and wavelength of incident radiation}

The design of a reliable water pollution optical sensor is fraught with challenges due to many parameters influencing the fidelity of the sensor that have to be determined. As a practical approach, we started our design process by using a genetic algorithm to find the optimal structural parameters of the single-layer Mg-based GMR structure for an analyte with the refractive index of 1.32 and TM polarization. The optimal parameters determined through this process were the duty cycle of the $\mathrm{MgO}$ grating, its thickness and period with values of $0.746 \mathrm{~nm}, 100 \mathrm{~nm}$ and $308.5 \mathrm{~nm}$, respectively.

To further characterize the structure, the diffraction efficiency of the reflected light was computed as a function of the thickness of the grating and the wavelength of the incident radiation in the vicinity of a resonance wavelength. Results are shown in Fig. 2 for the refractive indices of the analyte, 1.32 and 1.37, respectively. Furthermore, Figure 2 indicates that the TM and TE polarizations have different characteristics. It shows that for the TM polarized incident radiation, the spectral widths for most reflection peaks are narrower than that of the TE polarized one. However, in principle, one can design a filter for the TE polarized incident radiation 
with a comparably narrow resonance peak if the right grating thickness is chosen. For example, when we consider the TE mode spectral response for a grating thickness of $300 \mathrm{~nm}$ as shown in Fig. 2, the corresponding resonance peak is narrower.

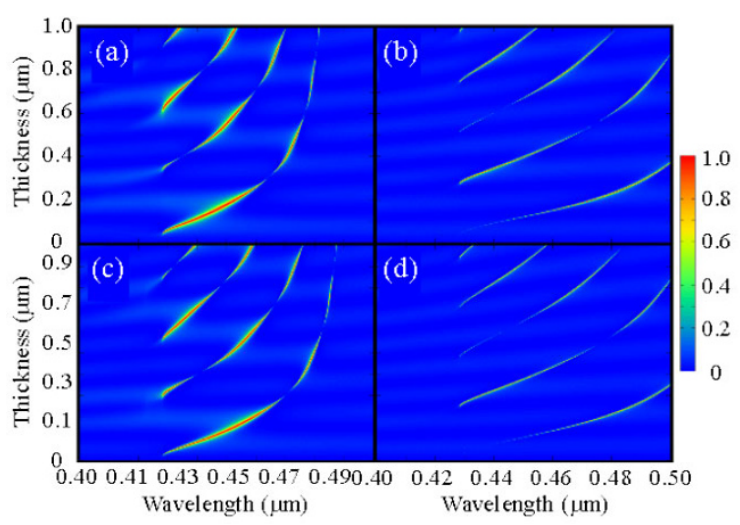

Fig. 2 Diffraction efficiency of the Mg-based GMR filter as a function of the incident wavelength and grating thickness for (a) and (c) TE polarized incident radiation; (b) and (d) TM polarized incident radiation [in the top subfigures (a)\&(b), the analyte refractive index is 1.32 while for the bottom ones (c)\&(d), it is 1.37].

Figure 3 shows the diffraction efficiency of both TM and TE polarized radiations, for a grating thickness of $100 \mathrm{~nm}$ and $300 \mathrm{~nm}$, respectively. The shallow grating has good optical characteristics of the low sidebands and narrow bandwidth for the TM polarized incident radiation, while the deep grating

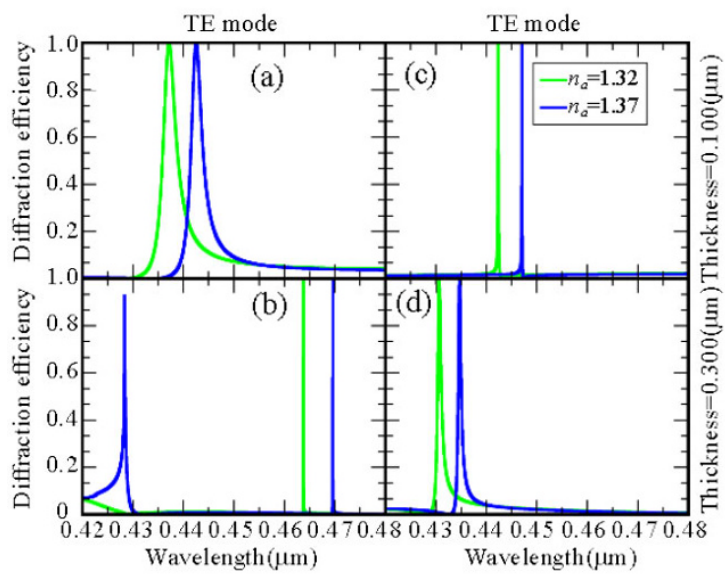

Fig. 3 Diffraction efficiency of the Mg-based GMR filter as the function of the incident wavelength for normal incidence for the grating thickness of $100 \mathrm{~nm}$ and $300 \mathrm{~nm}$, respectively: (a) and (b) for the TE mode and (c) and (d) for the TM mode. has the good optical characteristics and narrow bandwidth for the TE polarized incident radiation.

\subsection{Spectral sensitivity}

The spectral sensitivity is defined as the wavelength shift of the reflection peak per unit shift in the refractive index of the analyte (water), which is measured in the refractive index unit - RIU. This sensitivity was investigated for normal incidence in the violet region $(430 \mathrm{~nm}-460 \mathrm{~nm})$ of the visible spectrum for both TE and TM modes. Figure 3 displays the dependence of the diffraction efficiency of the $\mathrm{MgO} / \mathrm{MgF}_{2}$ GMR filter as the function of the incident wavelength for TE and TM modes, for two grating thicknesses, $100 \mathrm{~nm}$ (shallow grating) and $300 \mathrm{~nm}$ (deep grating). For the GMRF with the grating thickness of $100 \mathrm{~nm}$, the TE mode resonance peaks are located at $436.8 \mathrm{~nm}$ and $442.3 \mathrm{~nm}$ for the refractive indices of water, 1.32 and 1.37 , respectively. Accordingly, the spectral sensitivity of this mode is $110 \mathrm{~nm} / \mathrm{RIU}$, while the full-width at the half maximum (FWHM) is approximately $2.5 \mathrm{~nm}$. Similarly, for the TM mode, with the same grating thickness of $100 \mathrm{~nm}$, the resonance peaks occur at $442.2 \mathrm{~nm}$ and $447 \mathrm{~nm}$ for the refractive indices of water, 1.32 and 1.37, respectively. For this case, the spectral sensitivity is $96 \mathrm{~nm} / \mathrm{RIU}$, which is slightly smaller than that of the TE mode. However, the resonance peaks are much narrower with the FWHM less than $0.15 \mathrm{~nm}$, nearly 20 times smaller than that of the TE mode.

Unlike the grating with the grating thickness of $100 \mathrm{~nm}$, the TE polarized light in the 300-nm-thickness grating has resonance peaks in two locations that are different from the TM mode. The TE mode resonance peaks are extremely sharp with the smallest FWHM of $0.03 \mathrm{~nm}$ and located at $462.7 \mathrm{~nm}$ and $469.6 \mathrm{~nm}$ for the refractive indices of water, 1.32 and 1.37, respectively. The spectral sensitivity for this polarization mode is $138 \mathrm{~nm} / \mathrm{RIU}$. On the other hand, the TM polarized light resonance peaks are located at $430.6 \mathrm{~nm}$ and $434.7 \mathrm{~nm}$ for the 
refractive indices of water, 1.32 and 1.37, respectively. The spectral sensitivity and the FWHM are nearly $82 \mathrm{~nm} / \mathrm{RIU}$ and $0.84 \mathrm{~nm}$, respectively.

Figure 4 shows the variation of the diffraction efficiency with the wavelength of the incident radiation and refractive index of the analyte for TE and TM polarizations. This figure shows the following:

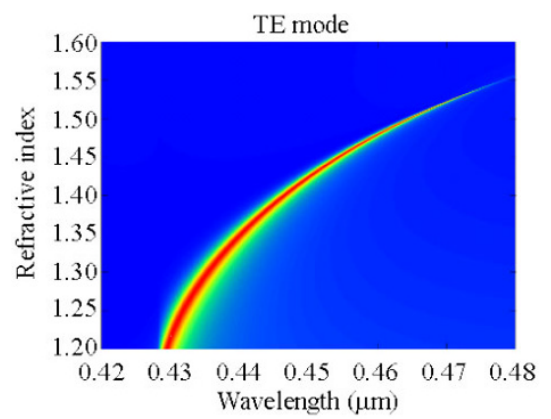

(a)

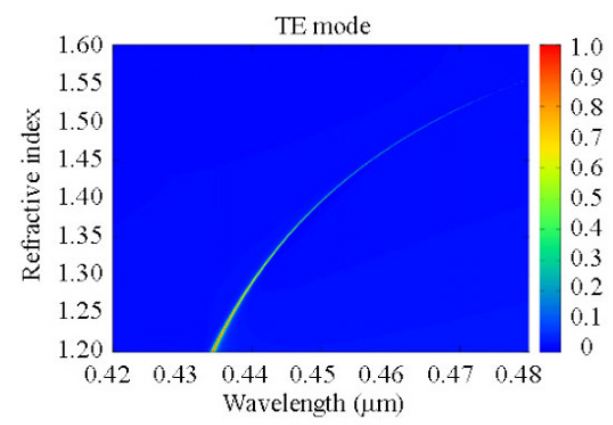

(b)

Fig. 4 Diffraction efficiency of the Mg-based GMR filter as the function of incident wavelength and refractive index for (a) TE and (b) TM mode, for the grating thickness of $100 \mathrm{~nm}$.

(1) The location of the resonance reflection peak depends nonlinearly and monotonically on the refractive index of the analyte. The larger the refractive index is, the longer the resonance wavelength is.

(2) The spectral sensitivity of the sensor also decreases as a function of the refractive index of the analyte.

(3) For both TM and TE polarization modes, the resonance peaks are narrower, and consequently their FWHMs are smaller for the larger refractive index values. Thus while a water pollution optical sensor can be designed to utilize the TE polarized incident radiation, this sensor will only have high fidelity at the relatively large refractive index of the analyte.

The spectral characterization of the GMRFs discussed above is summarized in Table 1. Table 1 shows that the TE mode spectral sensitivities are larger than those of their TM counterparts, with better performance of the deep grating, with the FWHM of $0.03 \mathrm{~nm}$ with respect to $2.5 \mathrm{~nm}$ in the case of the shallow grating.

Table 1 Grating thickness, polarization mode and spectral characterization of the GMRF used in this study.

\begin{tabular}{cccccc}
\hline $\begin{array}{c}\text { Polarization } \\
\text { mode }\end{array}$ & $\begin{array}{c}\text { Grating } \\
\text { thickness } \\
(\mathrm{nm})\end{array}$ & $\begin{array}{c}\text { Refractive } \\
\text { index of the } \\
\text { analyte }\end{array}$ & $\begin{array}{c}\text { Resonance } \\
\text { wavelength } \\
(\mathrm{nm})\end{array}$ & $\begin{array}{c}\text { FWHM } \\
(\mathrm{nm})\end{array}$ & $\begin{array}{c}\text { Sensitivity } \\
(\mathrm{nm} / \mathrm{RIU})\end{array}$ \\
\hline \multirow{2}{*}{$\mathrm{TM}$} & & 1.32 & 442.2 & 0.15 & 96.0 \\
& 100 & 1.37 & 447.0 & & \\
$\mathrm{TE}$ & & 1.32 & 436.8 & 2.5 & 110 \\
& & 1.37 & 442.3 & & \\
$\mathrm{TM}$ & & 1.32 & 430.6 & 0.84 & 82.0 \\
& 300 & 1.37 & 434.7 & & 138 \\
$\mathrm{TE}$ & & 1.32 & 462.7 & 0.03 & \\
\hline
\end{tabular}

\subsection{Angular sensitivity}

The angular sensitivity is defined as the angular shift in the resonance reflection maximum per unit shift in the refractive index of the analyte.

Figures 5(a) and 5(b) show the dependence of the diffraction efficiency of the $\mathrm{MgO} / \mathrm{MgF}_{2}$ GMR filter, with the grating thickness of $100 \mathrm{~nm}$ and $300 \mathrm{~nm}$, as a function of the incident angle for TE and TM polarized incident light sources at the wavelength of $632.8 \mathrm{~nm}$ (He-Ne gas laser).

According to Fig. 5, resonance peaks for the TE polarized laser for the sensor with the grating thickness of $100 \mathrm{~nm}$ are located at $27.22^{\circ}$ and $29.16^{\circ}$ for the refractive indices of water, 1.37 and 1.32 , respectively. The angular sensitivity is approximately 39 degrees/RIU in this mode, while the FWHM is nearly $0.26^{\circ}$. Figure $5(\mathrm{~b})$ indicates that the resonance peaks of the TE mode for the sensor with the grating thickness of $300 \mathrm{~nm}$ are located at 


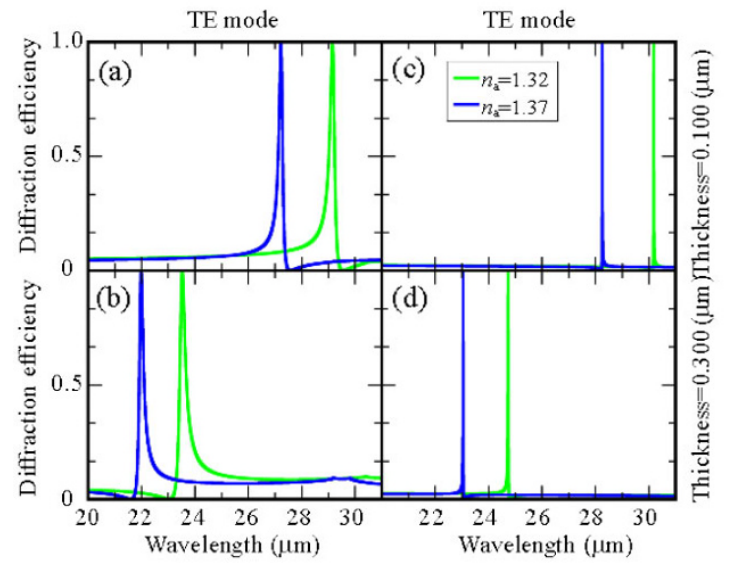

Fig. 5 Diffraction efficiency of the Mg-based GMR filter as a function of the incident angle at $\lambda=632.8 \mathrm{~nm}$ for the grating thickness of $100 \mathrm{~nm}$ and $300 \mathrm{~nm}$, respectively: (a) \& (b) for the TE mode and (c) \& (d) for the TM mode.

$21.99^{\circ}$ and $23.53^{\circ}$ for the refractive indices of water, 1.37 and 1.32 , respectively. For this sensor, the angular sensitivity is approximately 30.8 degrees/RIU with the FWHM of $0.19^{\circ}$. Finally, the dependence of the diffraction efficiency of the Mg-based GMR filter on the incident angle for the TM polarized incident radiation is given in Figs.5(c)-5(d). The TM mode resonance peaks for the sensor with a grating thickness of $100 \mathrm{~nm}$, are located at $28.27^{\circ}$ and $30.22^{\circ}$ for the refractive indices of water, 1.37 and 1.32 , respectively, leading to an angular sensitivity of 39 degrees/RIU with the FWHM of $0.014^{\circ}$. In contrast, the resonance peaks for the 300 -nm-thickness grating are located at $23.05^{\circ}$ and $24.73^{\circ}$ for the refractive indices of water, 1.37 and 1.32 , respectively, leading to an angular sensitivity of 33.6 degrees/RIU with the FWHM of $0.011^{\circ}$. Compared with the sensor with the grating thickness of $100 \mathrm{~nm}$ ( $\sim 39$ degrees/RIU), the sensor with the grating thickness of $300 \mathrm{~nm}(\sim 34$ degrees/RIU) has a lower angular sensitivity and a slightly wider FWHM. Evidently, both sensors are exhibiting similar sensitivity in the TM and TE polarization modes, but the FWHM is substantially larger (nearly 18 times) in the TE mode. The angular characterization of the GMRF discussed above is summarized in Table 2. Table 2 shows that although the sensitivities are nearly the same for both modes at each grating thickness, the TM mode is sharper than the TE mode for angular interrogation.

Table 2 Grating thickness, polarization mode and angular characterization of the GMRF used in this study.

\begin{tabular}{cccccc}
\hline $\begin{array}{c}\text { Polarization } \\
\text { mode }\end{array}$ & $\begin{array}{c}\text { Grating } \\
\text { thickness } \\
(\mathrm{nm})\end{array}$ & $\begin{array}{c}\text { Refractive } \\
\text { index of the } \\
\text { analyte }\end{array}$ & $\begin{array}{c}\text { Resonance } \\
\text { angle }\left(^{\circ}\right)\end{array}$ & $\begin{array}{c}\text { FWHM } \\
\left({ }^{\circ}\right)\end{array}$ & $\begin{array}{c}\text { Sensitivity } \\
(\text { degree/RIU) }\end{array}$ \\
\hline \multirow{2}{*}{$\mathrm{TM}$} & & 1.32 & 30.22 & 0.014 & 39.0 \\
& 100 & 1.37 & 28.27 & & \\
$\mathrm{TE}$ & & 1.32 & 29.16 & 0.26 & 39.0 \\
& & 1.37 & 27.22 & & \\
$\mathrm{TM}$ & \multirow{2}{*}{300} & 1.32 & 24.73 & 0.011 & 33.6 \\
& & 1.37 & 23.05 & & \\
$\mathrm{TE}$ & & 1.32 & 23.53 & 0.19 & 30.8 \\
\hline
\end{tabular}

\section{Conclusions}

A compact and easy to be fabricated Mg-based GMR filter was proposed to construct a high resolution water pollution sensor. Because of the dependence of the design of the GMR filter on variables such as the grating thickness, duty cycle, pitch and waveguide thickness as well as the refractive index of the analyte, we established the relationship between some of these variables and the reflected radiation intensity to guide the design process. Extensive structural parameters optimization yielded spectral and angular sensitivities better than $100 \mathrm{~nm} / \mathrm{RIU}$ and nearly 40 degrees/RIU, respectively. The spectral sensitivity of $138 \mathrm{~nm} / \mathrm{RIU}$ was obtained for normal incidence, in the TE mode with the deep grating with the thickness of $300 \mathrm{~nm}$ at $\lambda=462.7 \mathrm{~nm}$ with the FWHM of $0.03 \mathrm{~nm}$, while angular sensitivity of $39^{\circ} / \mathrm{RIU}$ was obtained for He-Ne laser, in the TM mode with the shallow grating with the thickness of $100 \mathrm{~nm}$, with the FWHM of $0.014^{\circ}$.

\section{Acknowledgment}

This work is supported by a grant from Birzeit University.

Open Access This article is distributed under the terms of the Creative Commons Attribution License which 
permits any use, distribution, and reproduction in any medium, provided the original author(s) and source are credited.

\section{References}

[1] Y. Fujiwara, H. Matsuda, K. Sato, M. Jimbo, and T. Kobayashi, "Magnetoresistance and electronic structure of granular films with $\mathrm{MgO}$ or $\mathrm{MgF}_{2}$ matrices," Journal of Physics: Conference Series, vol. 266, no. 1, pp. 012087, 2011.

[2] E. J. Denlinger, A. Fathy, D. Kalokitis, V. Pendrick, and B. Thaler, "High performance YBCO films," Report for 25 May-25 August 1994, pp. 1-13, 1994.

[3] S. S. Wang and R. Magnusson, "Theory and applications of guided-mode resonance filters," Applied Optics, vol. 32, no. 14, pp. 2606-2613, 1993.

[4] S. S. Wang, R. Magnusson, J. S. Bagby, and M. G. Moharam, "Guided-mode resonances in planar dielectric-layer diffraction gratings," Journal of the Optical Society of America A, vol. 7, no. 8, pp. 1470-1474, 1990.

[5] T. Clausnitzer, A. V. Tishchenko, E. Kley, H. Fuchs, D. Schelle, O. Parriaux, et al., "Narrowband, polarization-independent free-space wave notch filter," Journal of the Optical Society of America A, vol. 22, no. 12, pp. 2799-2803, 2005.

[6] N. Destouches, A. Tishchenko, J. Pommier, S. Reynaud, O. Parriaux, S. Tonchev, et al., " $99 \%$ efficiency measured in the -1 st order of a resonant grating," Optics Express, vol. 13, no. 9, pp. 3230-3235, 2005.

[7] S. S. Wang and R. Magnusson, "Multilayer waveguide-grating filters," Applied Optics, vol. 34, no. 14, p. 2414-2420, 1995.

[8] R. Magnusson and S. S. Wang, "New principle for optical filters," Applied Physics Letters, vol. 61, no. 9, pp. 1022-1024, 1992.

[9] M. S. Klimov, V. A. Sychugov, A. V. Tishchenko, and O. Parriaux, "Optimization of optical waveguide grating couplers," Fiber and Integrated Optics, vol. 11, no. 1, pp. 85-90, 1992.

[10] A. Sharon, D. Rosenblatt, A. A. Friesem, H. G. Weber, H. Engel, and R. Steingrueber, "Light modulation with resonant grating-waveguide structures," Optics Letters, vol. 21, no. 19, pp. 1564-1566, 1996.
[11] T. Katchalski, G. L. Yurista, A. Friesem, G. Martin, R. Hierle, and J. Zyss, "Light modulation with electro-optic polymer-based resonant grating waveguide structures," Optics Express, vol. 13, no. 12, pp. 4645-4650, 2005.

[12] B. Cunningham, P. Li, B. Lin, and J. Pepper, "Colorimetric resonant reflection as a direct biochemical assay technique," Sensors and Actuators B: Chemical, vol. 81, no. 2-3, pp. 316-328, 2002.

[13] I. Abdulhalim, "Biosensing configurations using guided wave resonant structures," in Optical waveguide sensing and imaging (proceedings of the NATO Advanced Study Institute on Optical Waveguide Sensing and Imaging in Medicine, Environment, Security and Defence, Gatineau, Québec, Canada, October 12-21, 2006), W. J. Bock, I. Gannot, and S. Tanev, Ed. Netherlands: Springer Netherlands, 2008, pp. 211-228.

[14] I. Abdulhalim, "Optimized guided mode resonant structure as thermooptic sensor and liquid crystal tunable filter," Chinese Optics Letters, vol. 7, no. 8, pp. 667-670, 2009.

[15] Y. Fang, A. M. Ferrie, N. H. Fontaine, J. Mauro, and J. Balakrishnan, "Resonant waveguide grating biosensor for living cell sensing," Biophysical Journal, vol. 91, no. 5, pp. 1925-1940, 2006.

[16] O. Parriaux and G. J. Veldhuis, "Normalized analysis for the sensitivity optimization of integrated optical evanescent-wave sensors," Journal of Lightwave Technology, vol. 16, no. 4, pp. 573-582, 1998.

[17] D. D. Wawro, S. Tibuleac, R. Magnusson, and H. Liu. "Optical fiber endface biosensor based on resonances in dielectric waveguide gratings," presented at BiOS 2000 The International Symposium on Biomedical Optics, San Jose, CA International Society for Optics and Photonics, January 22, 2000.

[18] R. E. Collin, Field theory of guided waves. New York: IEEE Press, 1991.

[19] M. G. Moharam, D. A. Pommet, E. B. Grann and T. K. Gaylord, "Stable implementation of the rigorous coupled-wave analysis for surface-relief gratings: enhanced transmittance matrix approach," Journal of the Optical Society of America A, vol. 12, no. 5, pp. 1077-1086, 1995.

[20] H. Rathge. "MRCWA - multilayer rigorous coupled wave analysis," 2010, available from: http://mrcwa. sourceforge.net.

[21] Filmetrics Inc., "Filmetrics index of refraction database," available from: http://www.filmetrics. com/refractive-index-database. 